\title{
Aktive Senioren senken Herztodrisiko um bis zu zwei Drittel
}

\author{
Wer über 65 Jahre alt ist und regelmäßig körperliche Aktivitäten von moderater \\ Intensität aufrechterhält, reduziert sein Herztodrisiko um mehr als $\mathbf{5 0} \%$, so das \\ Ergebnis einer finnischen Studie.
}

\begin{abstract}
_ Dass ein sportlicher Lebensstil vor Herz-Kreislauf-Erkrankungen schützt, ist hinlänglich bekannt und wissenschaftlich gut dokumentiert. Weniger klar ist die Datenlage bei älteren Leuten.

Aus diesem Grund hat eine Autorengruppe um Frau Prof. Riita Antikainen von der Universität Oulu in Finnland knapp 2.500 über 65-jährige Männer und Frauen für die „National FINRISK Study" rekrutiert und über im Median 11,8 Jahre beobachtet. Die Teilnehmer wurden nach ihren körperlichen Aktivitäten befragt und anhand der Antworten in drei Gruppen mit niedriger, moderater und hoher Aktivität eingeteilt.
\end{abstract}

Zudem wurden Risikofaktoren evaluiert. Herz- und tumorkranke Patienten wurden ausgeschlossen.

Im Beobachtungszeitraum starben 197 Teilnehmer aus kardiovaskulärer Ursache, bei 416 trat eine erste kardiovaskuläre Komplikation auf. Nach Korrektur für kardiovaskuläre Risiko- und soziale Faktoren zeigte sich, dass Personen mit moderater bzw. hoher körperlicher Aktivität gegenüber körperlich Untätigen ein um 31 bzw. 45 \% geringeres Risiko für eine akute kardiovaskuläre Komplikation aufwiesen. Die kardiovaskuläre Sterblichkeit war gar um 54 bzw. $66 \%$ geringer.
Sich regelmäßig körperlich zu betätigen, wenn man über 65 Jahre alt ist, kann eine Herausforderung sein, so Antikainen. Die Studienergebnisse sollten aber Motivation sein. Denn fitte Senioren reduzieren ihr Herzinfarkt- und Schlaganfall-Risiko deutlich.

Als moderate Aktivität galten übrigens schon Spazieren gehen, radeln, gärtnern, fischen oder jagen. Als hohe Aktivität eingestuft wurde anstrengende Gartenarbeiten, Schwimmen, Joggen, Gymnastik, Skifahren und Ballspiele. -

Dr. Dirk Einecke

- Jahrestagung der European Society of Cardiology (ESC), Rom, 27.-31. August 2016

\section{Glosse}

\section{Auf Tuchfühlung mit Experten für Herz und Seele}

\begin{abstract}
— Kongresse sind dazu da, dass man viele Leute trifft. Ansonsten könnte man ja zu Hause bleiben und alles am Bildschirm verfolgen. Das wäre einfacher und billiger. Damit man auf solchen Veranstaltungen aber auch zueinander findet, haben sich die Veranstalter die „Meet the..."- Sessions einfallen lassen.

Beim diesjährigen europäischen Kardiologenkongress war sicherlich das "Meet the Pope" der eigentliche Höhepunkt. Der Papst hatte sich aber ein besonderes Format für seinen Auftritt ausbedungen. Er sprach im Freien und das ansonsten nach Vorträgen übliche Frage-und-Antwort-Spiel war nicht vorgesehen. Eigentlich schade; denn gerne hätte man ihn um eine Erklärung gebeten, warum die Kardiologen trotz intensiver Bemühungen mittels moderner
\end{abstract}

bildgebender Diagnostik bisher die Seele nicht im Herzen haben finden können. Aber wo soll sie denn sonst sein?

Dann gab es noch das „Meet the legends", also das Treffen mit den Päpsten der Kardiologie. Diese in der Tat legendären Persönlichkeiten haben die Kardiologie schon in einer Zeit erlebt, als das Stethoskop noch der einzige Zugang zum Herzen war. Sehr interessant und unterhaltsam. Leider gab es für die Teilnahme aber keine Fortbildungspunkte.

Üblich sind mittlerweile auch Kontakte über den Tellerrand des eignen Fachgebietes hinaus: Cardiology meets Nephrology or Diabetolgy or Rheumatology etc., also jeder trifft jeden und am Ende weiß man oft gar nicht mehr, wer für welches Fachgebiet steht. Interdisziplinarität ist eben gefragt. Erfreulich war, dass sich bei kontroversen Debatten die Kombattanten stets die körperliche Unversehrtheit garantieren konnten.

Am spannendsten sind die "Meet the Experts", also das Treffen mit echten Experten und solchen, die sich dafür halten. Die Zahl letzterer steigt mit der Zahl der ungelösten Probleme. Manchmal drängt sich die Frage auf, wer eigentlich zuerst da war, die Fragestellung oder der Experte. Ähnlich wie bei der Henne und dem Ei. Nach dem Motto „Eine Lösung sucht ihr Problem" wurden dort überzeugende Daten präsentiert zu Fragen, die ein Schmalspurkardiologe gar nicht stellen würde. Doch wer vieles bringt, wird manchem etwas bieten, und jeder geht zufrieden aus dem Haus. So lautet das Fazit des Kongresses: „Nice to meet you“.

Dr. Peter Stiefelhagen 